Conclusions The Apollo OverStitch system can be used in the management of esophageal leaks and has proven efficacy and safety. Although this technique does require expertise, it provides a successful minimally invasive method that can help in the management of these often critically ill patients. This is the first reported case series of successful utilisation of the Apollo OverStitch System in combination with a stent in the management of esophageal leaks.

\section{PTH-035 EUS-GUIDED BILIARY AND ENDOLUMINAL DRAINAGE BY LAMS: INITIAL EXPERIENCE FROM A HPB/ TRANSPLANT CENTRE}

Geri Keane*, David Reffitt, Philip Harrison, Deepak Joshi, John Devlin. Kings College Hospital, London, UK

\subsection{6/gutjnl-2019-BSGAbstracts.60}

Introduction Endoscopic ultrasound-guided biliary or enteral drainage is an emerging alternative to managing biliary or enteral obstruction, when endoscopic retrograde cholangiopancreatography (ERCP) or duodenal stenting fail. Recently developed electrocautery-enhanced Lumen-Apposing Metal Stents (LAMS), allow endoscopic anastomosis (Hot AXIOS ${ }^{\mathrm{TM}}$ system, Boston Scientific) to facilitate EUS guided choledochojejunostomy or gastrojejunostomy. EUS guided hepaticogastrostomy is also feasible; using a dedicated half covered biliary SEMS (Giobor ${ }^{\circledR}$ stent). The aim of this study was to assess the safety and effectiveness of these stents in gallbladder, biliary and endoluminal drainage where standard approaches have failed.

Methods Case series of EUS guided biliary or enteral drainage from a tertiary referral HPB/Liver Transplant centre between January 2017 and January 2019.

Results During the 2 year study period, 19 cases were undertaken on 14 patients; 7 Male, 7 Female. Median age 77 (range 34-91). 10 of the patients had malignant disease, 4 benign. Indications included 6 EUS guided biliary drainage with LAMS from D1, 4 EUS guided gallbladder drainage, 3 EUS wire guided rendezvous, 2 EUS guided drainage of an obstructed hepaticojejunostomy, 2 EUS guided gastrojejunostomy, 1 gastrojejunostomy stent change, 1 hepaticogastrostomy. The procedure was clinically successful in $68 \%(13 / 19)$ of cases. Median length of stay post procedure was 4 days (range 1-32). Adverse events occurred in 32\% (6/19), 5/6 were small bile leaks, which settled clinically without need for further intervention, one case of stent displacement requiring surgical removal.

Conclusion In our experience, EUS-guided LAMS placement for novel applications, performed by experienced endosonographers was safe and feasible in selected cases of benign and malignant disease.

\section{PTH-036 INTRA-DUCTAL COVERED SELF-EXPANDING METAL STENTS ARE NOT JUST FOR THE MANAGEMENT OF POST-TRANSPLANT ANASTOMOTIC STRICTURES}

${ }^{1}$ Geri Keane*, ${ }^{2}$ Ben Warner, 'David Reffitt, ${ }^{1}$ John Devlin, ${ }^{1}$ Phillip Harrison, ${ }^{1}$ Deepak Joshi. ${ }^{1}$ Kings College Hospital, London, UK; ${ }^{2}$ Dartford NHS Trust, Dartford, Kent

\subsection{6/gutjnl-2019-BSGAbstracts.61}

Introduction Traditional fully covered self-expanding metal stents (FCSEMS) can have poor durability in certain situations e.g. post transplant anastomotic strictures, leading to proximal or distal stent migration. Novel intra-ductal FCSEMS have been developed with an antimigration waist, short stent length and removal wires that are deployed in the duodenum to allow easy removal. These stents have improved the management of post-transplant anastomotic strictures, substantially decreasing the number of ERCPs these patients require and are now becoming the preferred stent in this situation in a number of transplant centres globally. Non-transplant indications for this stent have rarely been described.

Methods Case series of novel indications for ID-FCSEMS from a tertiary referral HPB/Liver Transplant centre.

Results 10 ID-FCSEMS were inserted in 9 patients for a range of indications (bile leak following transplant (3) or hepatectomy (1), bile leak following spontaneous perforation of the bile duct during acute pancreatitis, stricturing due to bile duct trauma post laparoscopic cholecystectomy (2), Mirizzi syndrome or choledochal varices). Five stents were inserted into the $\mathrm{CHD} / \mathrm{CBD}$ and $5 / 10$ into the proximal IHD/hilum. Median age of the patients was 51 years, and the majority were female (55\%). Five patients had had a previous ERCP and plastic stent, which had been unsuccessful. 90\% (9/10) of the stents inserted were $8 \times 40 \mathrm{~mm}$, with a single $10 \times 40 \mathrm{~mm}$ stent inserted in one case. A sphincterotomy was performed in $60 \%$ cases and $30 \%$ of strictures were balloon dilated prior to stent insertion. Clinical resolution of the leak or stricture was seen in $100 \%$ of cases. There were no associated episodes of stent migration or post ERCP pancreatitis. All stents were removed without any difficulty. No patients required a further ERCP or intervention following ERCP with ID-FCSEMS insertion.

Conclusion This novel ID-FCSEMS is associated with high rate of clinical success in a range of clinical indications, over and above the management of post-transplant anastomotic strictures. There were no associated adverse events. As a result, there are growing number of applications for this novel stent.

\section{PTH-037 BURIED PEG BUMPER SYNDROME CAN NEARLY ALWAYS BE MANAGED ENDOSCOPICALLY: A 14 YEAR PERSONAL SERIES}

${ }^{1}$ Gireesh Kothegal Marimahadevappa*, ${ }^{2} \mathrm{Sr}$ Mandy Parry, ${ }^{2}$ Tamsin Boyce, ${ }^{2}$ Miles Allison. ${ }^{1} A B M U H B$, Swansea, UK; ${ }^{2} A B \cup H B$, Newport, UK

\subsection{6/gutjnl-2019-BSGAbstracts.62}

Introduction Most patients needing Percutaneous Endoscopic Gastrostomy (PEG) tube feeding have incurable neurological disease, often with behavioural difficulty. Patient traction on tubing and weight gain predispose to the internal PEG bumper eroding through the anterior gastric wall and becoming buried, leading to the condition generally known as Buried Bumper Syndrome (BBS). This could be challenging to deal with as it is associated with complex decision making process. Methods We collected the data of the patients who had BBS between 2004 to 2018, from two sites in Aneurin Bevan University Health Board (AB UHB). All patients were managed by one endoscopist across the sites. This included patients who had their procedures done both under sedation and general anaesthesia. Data reviewed included the endoscopic techniques used, sedation practice, new PEG tube placements, complications, limitations and outcome of these procedures 